\title{
Posterior Uveitis Secondary to Syphilis
}

\author{
Gaurav Gupta, MD and Dmitry Pyatetsky, MD
}

\begin{abstract}
A
24-year-old man presented to the ophthalmologist complaining of decreased vision and intermittent dull pain in the left eye of 5 -months duration. Ophthalmologic examination revealed visual acuity of 20/70, mild inflammatory reaction in the anterior chamber, vitreous haze (blur in figure 1), optic nerve edema, and retinal arteriolar narrowing and sheathing (figure 1). Additional history was significant for recurrent facial zoster, anal condyloma acuminatum, and a recent episode of pneumonia. General examination revealed multiple umbilicated lesions on facial skin consistent with a diagnosis of molluscum contagiosum. There was a fading macular rash on the palms of his hands and the soles of his feet. The differential diagnosis for this combination of long-standing vitritis and retinal arteritis in the setting of multiple comorbidities suggestive of human immunodeficiency virus (HIV) is highly suspicious for syphilis, although it also includes necrotizing herpetic retinopathies, toxoplasmosis retinochoroiditis and non-infectious etiologies such as sarcoidosis. ${ }^{1,2}$ Serological testing confirmed the clinical diagnosis of tertiary syphilis and HIV. The eye pain and vitritis responded to intravenous penicillin $\mathrm{G}$, however the vision remained unchanged.
\end{abstract}

\section{References}

1. Gaudio PA. Update on ocular syphilis. Curr Opin Ophthalmol 2006;17: $562-566$

2. Chao JR, Khurana RN, Fawzi AA, Reddy HS, Rao NA. Syphilis: reemergence of an old adversary. Ophthalmology 2006;113: 2074-2079.

Figure 1 . Examination of the fundus shows evidence of long-standing vitritis and retinal arteritis as evidenced by the presence of (a) mild inflammation, optic nerve edema (arrow) and (b) vitreous haziness and presence of retinal arteriolar narrowing and sheathing (arrow).

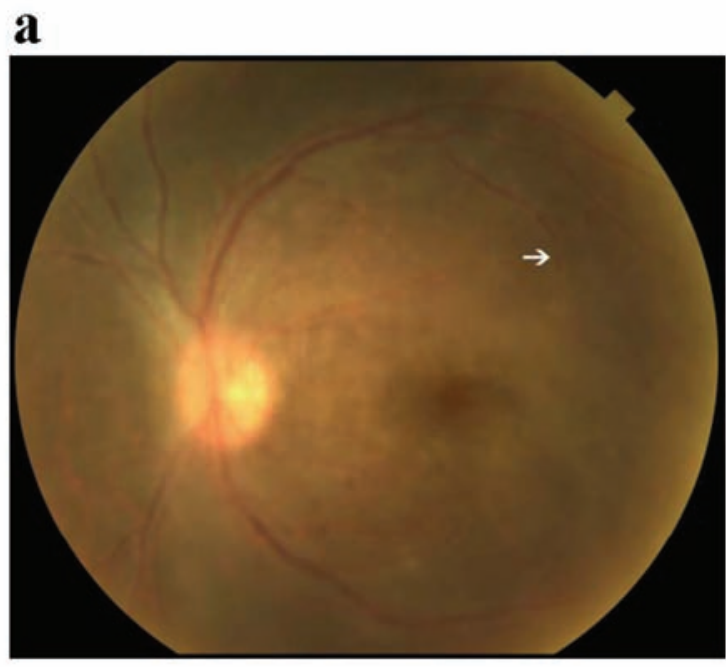

b

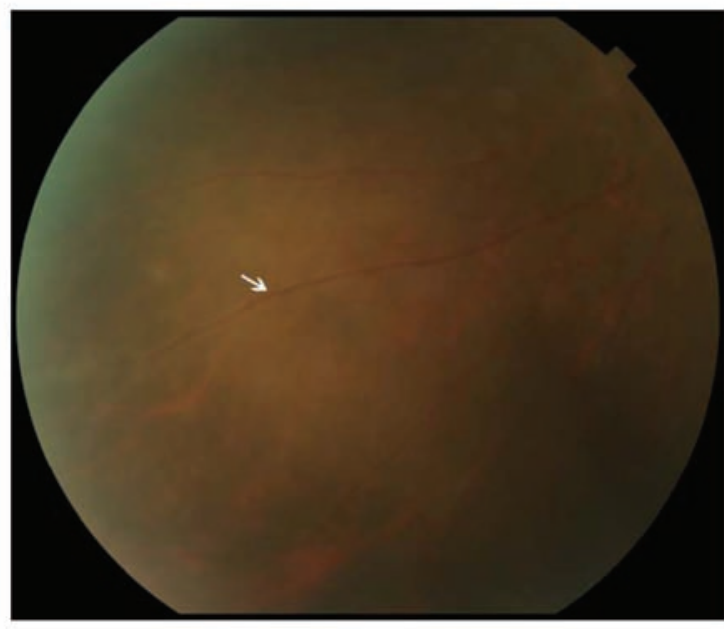

Author Affiliations:

Gaurav Gupta, MD*

Department of Ophthalmology, Marshfield Clinic

1000 North Oak Avenue, Marshfield,WI 54449 USA

Email: gupta.gaurav@marshfieldclinic.org and

Department of Ophthalmology, Northwestern University

Chicago, IL 606II USA

Dmitry Pyatetsky, MD

Department of Ophthalmology, Northwestern University

Chicago, IL 606II USA

* Corresponding Author
Keywords: Syphilis; Uveitis;Vessel sheathing

Received: November 10, 2008

Accepted: December 10, 2008

doi: $10.3|2| / \mathrm{cmr} .2009 .832$

Th: Aperture, like the opening in the lens of a microscope that allows light to pass through, is a forum for art, humor, and images that provides a portal for new or different views of medicine and research. 